\title{
TREINAMENTO DE FORÇA PRECOCE EM TERAPIA INTENSIVA NA INSUFICIÊNCIA CARDÍACA:MOBILIZAÇÃO PRECOCE
}

\author{
EARLY STRENGTH TRAINING IN INTENSIVE CARE IN HEART FAILURE: EARLY \\ MOBILIZATION
}

\author{
Beatriz Souza Nascimento de Jesus ${ }^{1}$ \\ Sanayara Leite Eufrásio ${ }^{2}$ \\ Lara Maria Fernandes Pereira ${ }^{3}$ \\ Cínthia Figueiredo Córdova da Costa ${ }^{4}$ \\ Diana FerreiraPacheco ${ }^{5}$
}

RESUMO: A insuficiência cardíaca (IC) é uma complexa síndrome clínica de diversas etiologias e com elevada prevalência, caracterizada por falência no suprimento adequado de sangue em relação ao retorno venoso e às necessidades metabólicas do indivíduo, cursando com intolerância ao esforço físico, consequentemente, diminuindo a capacidade funcional. $\mathrm{O}$ objetivo deste estudo consiste em comprovar a eficácia da mobilização precoce em pacientes com IC no leito, pelo fisioterapeuta, e ressaltar os benefícios dos exercícios físicos e a melhora na qualidade de vida. Foram realizadas pesquisas bibliográficas nas bases de dados PubMed, SciELO, ProQuest e PEDro, onde foram consultados io artigos publicados entre os anos 2009 a 2020. Foram utilizados os seguintes descritores para a busca: Mobilização precoce, Fisioterapia na UTI (Unidade de Terapia Intensiva), Insuficiência cardíaca, Cardiopatias, Treinamento de força e Mobilização precoce na UTI. Dos estudos analisados, os pacientes apresentaram melhora do desempenho funcional, marcha, espasticidade e também na qualidade de vida. A mobilização precoce por meio do fisioterapeuta atua diretamente na reabilitação desses pacientes promovendo ganhos significativos, contribuindo assim de forma bastante eficaz para a sua qualidade de vida.

Palavras-chaves: Fisioterapia na UTI. Insuficiência cardíaca. Cardiopatias. Treinamento de força.

ABSTRACT: Heart failure (HF) is a complex clinical syndrome of different etiologies and with high prevalence, characterized by failure in the adequate blood supply in relation to the venous return and the individual's metabolic needs, resulting in intolerance to physical effort, consequently, decreasing thefunctional capacity. The aim of this study is to prove the effectiveness of early mobilization in patients with HF in bed, by the physiotherapist, and to highlight the benefits of physical exercises and the improvement in quality of life. Bibliographic searches were

\footnotetext{
${ }^{1}$ Graduada em Fisioterapia. Instituição: UNICEPLAC - Centro Universitário do Planalto Central Apparecido dos Santos.E-mail: biasz33@gmail.com.

${ }^{2}$ Graduanda em Fisioterapia. Instituição: UNICEPLAC - Centro Universitário do Planalto Central Apparecido dos Santos.

${ }^{3}$ Graduanda em Fisioterapia. Instituição: UNICEPLAC - Centro Universitário do Planalto Central Apparecido dos Santos.

${ }^{4}$ Graduada em Fisioterapia. Instituição: UNICEPLAC - Centro Universitário do Planalto Central Apparecido dos Santos.

${ }^{5}$ Docente do curso de Fisioterapia do UNICEPLAC - Centro Universitário do Planalto CentralApparecido dos Santos. E-mail: diana.pacheco@uniceplac.edu.br.
} 
carried out in the PubMed, SciELO, ProQuest and PEDro databases, where ro articles published between the years 2009 to 2020 were consulted. The following descriptors were used for the search: Early mobilization, Physiotherapy in the ICU, Heart failure, Heart disease, Strength training and Early mobilization in the ICU. From the studies analyzed, patients showed improvement in functional performance, gait, spasticity and also in quality of life. Early mobilization by means of a physical therapist acts directly in the rehabilitation of these patients, promoting significant gains, thus contributing quite effectively to their quality of life.

Keyword: Physiotherapy in the ICU. Cardiac insufficiency. Heart diseases. Strength training.

\section{INTRODUÇÃO}

O aumento da prevalência de doenças crônicas degenerativas não transmissíveis é provocado pelo envelhecimento populacional. Dentro desse contexto, há insuficiência cardíaca (IC), destacada pelo aumento dos seus casos a cada década de vida, sendo uma das principais agentes de hospitalização.

A IC é uma complexa síndrome clínica de diversas etiologias e com elevada prevalência, caracterizada por falência no suprimento adequado de sangue em relação ao retorno venoso e às necessidades metabólicas do indivíduo, cursando com intolerância ao esforço físico, consequentemente, diminuindo a capacidade funcional (BARRETO \& RAMIRES, 1998).

A diminuição da capacidade funcional acarreta expressiva redução da qualidade de vida dos pacientes portadores de IC na medida em que os sintomas de dispneia, edemas, cansaço fácil e intolerância à atividade aparecem, tornam-se mais presentes, induzindo o paciente à inatividade físicacomo forma de preservar energia e evitar os sintomas, responsável por pesado consumo de meios assistenciais, que atinge, sobretudo, idosos, podendo ser tratada de várias formas entre elas a reabilitação cardíaca (FARIAS \& SANTOS, 2000).

A mobilização precoce em paciente com IC em UTI tem sido bastante estudada e ultimamentepraticada de maneira ainda discreta, porém vem crescendo a cultura e os estudos em relação a este tema uma vez que a imobilização no leito é causa de inúmeros fatores de risco e para o agravamento do quadro clínico não só de pacientes com IC, mas também com outras morbidades. A mobilização precoce, ainda no ambiente de UTI, tem se mostrado eficaz para a recuperação e diminuição no tempode internação, além de trazer benefícios para o organismo e bem estar de pacientes, como: aumento de força muscular periférica e da pressão inspiratória máxima, menor número de dias de internação, além de melhorar a funcionalidade pós-alta (DANTAS et al, 2012).

Os estudos nos mostram que a reabilitação cardíaca (RC) de maneira precoce deve ser 
incluída como opção de tratamento da doença cardiovascular, contribuindo para uma melhor qualidade de vida do paciente com IC. Os efeitos benéficos dos exercícios físicos são vários dentre eles melhoria da função cardiovascular e respiratória, melhora da capacidade funcional e qualidade de vida.

O objetivo deste estudo consiste em comprovar a eficácia da mobilização precoce em pacientes com IC no leito, pelo fisioterapeuta, e ressaltar os benefícios dos exercícios físicos e a melhora na qualidade de vida.

\section{MATERIAL E MÉTODOS}

Trata-se de um levantamento de dados encontrados na literatura. Foram realizadas pesquisas bibliográficas, nas bases de dados PubMed, SciELO, ProQuest e PEDro, onde foram consultados cerca de 30 artigos e apenas io foram selecionados. Foram lidos e analisados os artigos encontrados na área de fisioterapia que realizaram algum tratamento voltado para pacientes com IC. Foram incluídos artigos com texto completo disponível, em português ou inglês. Os seguintes descritores para a busca foram utilizados: Mobilização precoce, Fisioterapia na UTI, Insuficiência cardíaca, Cardiopatias, Treinamento de força e Mobilização precoce na UTI.

Os critérios de inclusão foram artigos publicados entre 2009 a 2020, com os descritores acimamencionados. O período da busca foi de fevereiro a abril de 2020. Foram excluídos artigos que não atenderam os critérios acima especificados.

\section{RESULTADOS E DISCUSSÃO}

Após a busca foi realizado fichamento de todos os artigos, de acordo com título, autores/tipo, objetivo,base de dados, descritores utilizados e achados.

Quadro I Características dos estudos selecionados 2009-2020

\begin{tabular}{|l|c|c|c|c|c|}
\hline Título & Autore & Objetivo & Base & Descrito & Achados \\
& s/ & & sde & res & \\
& Tipos & & $\mathrm{dad}$ & utilizad & \\
& & & os & os & \\
\hline
\end{tabular}




\begin{tabular}{|c|c|c|c|c|c|}
\hline \begin{tabular}{|l|} 
mpacto da \\
eambulaçã \\
associada à \\
hobilização \\
recoce em \\
acientes \\
ríticos
\end{tabular} & $\begin{array}{l}\text { Fonseca et } \\
\text { al } \\
\text { (Revisã } \\
\text { o } \\
\text { Sistem } \\
\text { ática) }\end{array}$ & $\begin{array}{l}\text { Verificar na } \\
\text { literatura o } \\
\text { impactoda } \\
\text { deambulaçã } \\
\text { o associada } \\
\text { à } \\
\text { mobilização } \\
\text { precoce em } \\
\text { pacientes } \\
\text { críticos } \\
\text { internados } \\
\text { em UTI. }\end{array}$ & $\begin{array}{l}\text { ProQu } \\
\text { est }\end{array}$ & $\begin{array}{l}\text { Mobilização } \\
\text { precoce; } \\
\text { Deambulação; } \\
\text { Unidade de terapia } \\
\text { intensiva; } \\
\text { Mortalidade } \\
\text { hospitalar. }\end{array}$ & $\begin{array}{l}\text { A } \\
\text { deambulaç } \\
\text { ão } \\
\text { impactou } \\
\text { positivame } \\
\text { nte no } \\
\text { tempo de } \\
\text { internação } \\
\text { hospitalar, } \\
\text { de UTIe } \\
\text { de } \\
\text { ventilação } \\
\text { mecânica } \\
\text { em } \\
\text { pacientes } \\
\text { graves. }\end{array}$ \\
\hline $\begin{array}{l}\text { Capacida } \\
\text { de } \\
\text { funcional } \\
\text { como } \\
\text { preditor } \\
\text { de } \\
\text { qualidade } \\
\text { de vida } \\
\text { na } \\
\text { insuficiê } \\
\text { ncia }\end{array}$ & $\begin{array}{l}\text { Ulbrich et } \\
\text { al } \\
\text { (Estud } \\
\text { o } \\
\text { transv } \\
\text { ersal } \\
\text { descrit } \\
\text { ivo) }\end{array}$ & $\begin{array}{l}\text { Determinar } \\
\text { a relação } \\
\text { dos } \\
\text { domínios da } \\
\text { QV com a } \\
\text { CF de } \\
\text { pacientes } \\
\text { com IC, } \\
\text { assim como } \\
\text { propor } \\
\text { pontos de }\end{array}$ & $\begin{array}{l}\text { SciEL } \\
\text { O }\end{array}$ & $\begin{array}{l}\text { Reabilitação } \\
\text { cardiopulmonar e } \\
\text { metabólica; } \\
\text { Disfunção } \\
\text { ventricular; Estilo } \\
\text { de vida. }\end{array}$ & $\begin{array}{l}\text { Os } \\
\text { portadores } \\
\text { de IC com } \\
\text { mais de } \\
\text { três meses } \\
\text { de RCPM } \\
\text { apresentar } \\
\text { am } \\
\text { melhor } \\
\text { QV e } \\
\text { capacidade }\end{array}$ \\
\hline
\end{tabular}




\begin{tabular}{|l|l|l|l|}
\hline cardíaca & $\begin{array}{l}\text { corte dos } \\
\text { domínios da } \\
\text { QV por } \\
\text { meio da CF. }\end{array}$ & & funcional \\
& do que os \\
& iniciantes. \\
& O \\
& domínio \\
& emocional \\
& do \\
& questionár \\
& io de \\
& Minnesota \\
&
\end{tabular}

\begin{tabular}{|c|c|c|c|c|c|}
\hline & & & & & $\begin{array}{l}\text { preditor de } \\
\text { pontode } \\
\text { corte da } \\
\text { capacidade } \\
\text { funcional. }\end{array}$ \\
\hline $\begin{array}{l}\text { Fisioterapia } \\
\text { motora em } \\
\text { pacientes } \\
\text { adultos em } \\
\text { terapia } \\
\text { intensiva }\end{array}$ & $\begin{array}{l}\text { Borges et al } \\
\text { (Artig } \\
\text { o de } \\
\text { Revisã } \\
\text { o) }\end{array}$ & $\begin{array}{l}\text { Realizar uma } \\
\text { revisão da } \\
\text { literatura } \\
\text { abordando o } \\
\text { tema } \\
\text { fisioterapia } \\
\text { motora para } \\
\text { pacientes } \\
\text { adultos em } \\
\text { unidades de }\end{array}$ & SciELO & $\begin{array}{l}\text { Modalidades de } \\
\text { fisioterapia; Adulto; } \\
\text { Cuidados } \\
\text { Intensivos. }\end{array}$ & $\begin{array}{l}\text { Mais estudos } \\
\text { se fazem } \\
\text { necessário } \\
\text { para se } \\
\text { identificaro } \\
\text { tipo de } \\
\text { exercício, } \\
\text { duração, } \\
\text { intensidade e } \\
\text { a }\end{array}$ \\
\hline
\end{tabular}




\begin{tabular}{|c|c|c|c|c|c|}
\hline & & $\begin{array}{l}\text { terapia } \\
\text { intensiva. }\end{array}$ & & & $\begin{array}{l}\text { repercussão } \\
\text { da } \\
\text { fisioterapia } \\
\text { motora } \\
\text { precoce em } \\
\text { grupos } \\
\text { específicos } \\
\text { de } \\
\text { pacientes. }\end{array}$ \\
\hline $\begin{array}{l}\text { Segurança } \\
\text { e Eficácia } \\
\text { do } \\
\text { Treinamen } \\
\text { to Aeróbio } \\
\text { Combinad } \\
\text { o À } \\
\text { Ventilação } \\
\text { Não- } \\
\text { Invasiva } \\
\text { em } \\
\text { Pacientes } \\
\text { com } \\
\text { Insuficiênc } \\
\text { ia } \\
\text { Cardíaca } \\
\text { Aguda }\end{array}$ & $\begin{array}{l}\text { Oliveira } \\
\text { et al } \\
\text { (Estudo } \\
\text { Randomiza } \\
\text { do) }\end{array}$ & $\begin{array}{l}\text { Investigar os } \\
\text { efeitos do } \\
\text { exercício } \\
\text { físico isolado } \\
\text { ou associado à } \\
\text { ventilação } \\
\text { não- }\end{array}$ & $\begin{array}{l}\text { SciEL } \\
\text { O } \\
\text { PubM } \\
\text { ed }\end{array}$ & $\begin{array}{l}\text { Exercício; } \\
\text { Insuficiência } \\
\text { Cardíaca Aguda; } \\
\text { Ventilação Não } \\
\text { Invasiva; } \\
\text { Fisioterapia; } \\
\text { Reabilitação. }\end{array}$ & $\begin{array}{l}\text { O exercício } \\
\text { físico foi } \\
\text { seguro em } \\
\text { pacientes } \\
\text { com IC } \\
\text { aguda, não } \\
\text { houve } \\
\text { complicaçõe } \\
\text { s } \\
\text { hospitalares } \\
\text { e, }\end{array}$ \\
\hline
\end{tabular}




\begin{tabular}{|c|c|c|c|c|c|}
\hline $\begin{array}{l}\text { Efeitos da } \\
\text { mobilizaçã } \\
\text { o precoce } \\
\text { em } \\
\text { pacientes } \\
\text { críticos } \\
\text { internados } \\
\text { emUTI }\end{array}$ & $\begin{array}{l}\text { Isnanda } \\
\text { Tarciara } \\
\text { Silvae } \\
\text { Alinne } \\
\text { Alvez } \\
\text { Oliveira } \\
\text { (Artigo de } \\
\text { Revisão) }\end{array}$ & $\begin{array}{l}\text { Investigar os } \\
\text { efeitos da } \\
\text { mobilização } \\
\text { precoce em } \\
\text { pacientes } \\
\text { críticos } \\
\text { internados em } \\
\text { UTI, } \\
\text { identificando } \\
\text { quais as } \\
\text { técnicas de } \\
\text { mobilização } \\
\text { precoce mais } \\
\text { utilizadas } \\
\text { pelos } \\
\text { fisioterapeuta } \\
\text { s neste setor. }\end{array}$ & SciELO & $\begin{array}{l}\text { Mobilização } \\
\text { Precoce; } \\
\text { Fisioterapia; } \\
\text { Unidade de Terapia } \\
\text { Intensiva. }\end{array}$ & $\begin{array}{l}\text { As técnicas } \\
\text { executadas } \\
\text { pelos } \\
\text { fisioterapeu } \\
\text { tas } \\
\text { trouxeram } \\
\text { muitos } \\
\text { benefícios } \\
\text { aos } \\
\text { participante } \\
\text { s dos } \\
\text { ensaios, } \\
\text { como } \\
\text { aumentode } \\
\text { força } \\
\text { muscular } \\
\text { periférica, } \\
\text { menor } \\
\text { número } \\
\text { de dias de }\end{array}$ \\
\hline
\end{tabular}

\begin{tabular}{|l|l|l|l|l|}
\hline & & & & internação e \\
& & & & melhor \\
& & & & funcionalid \\
ade \\
pós-alta.
\end{tabular}




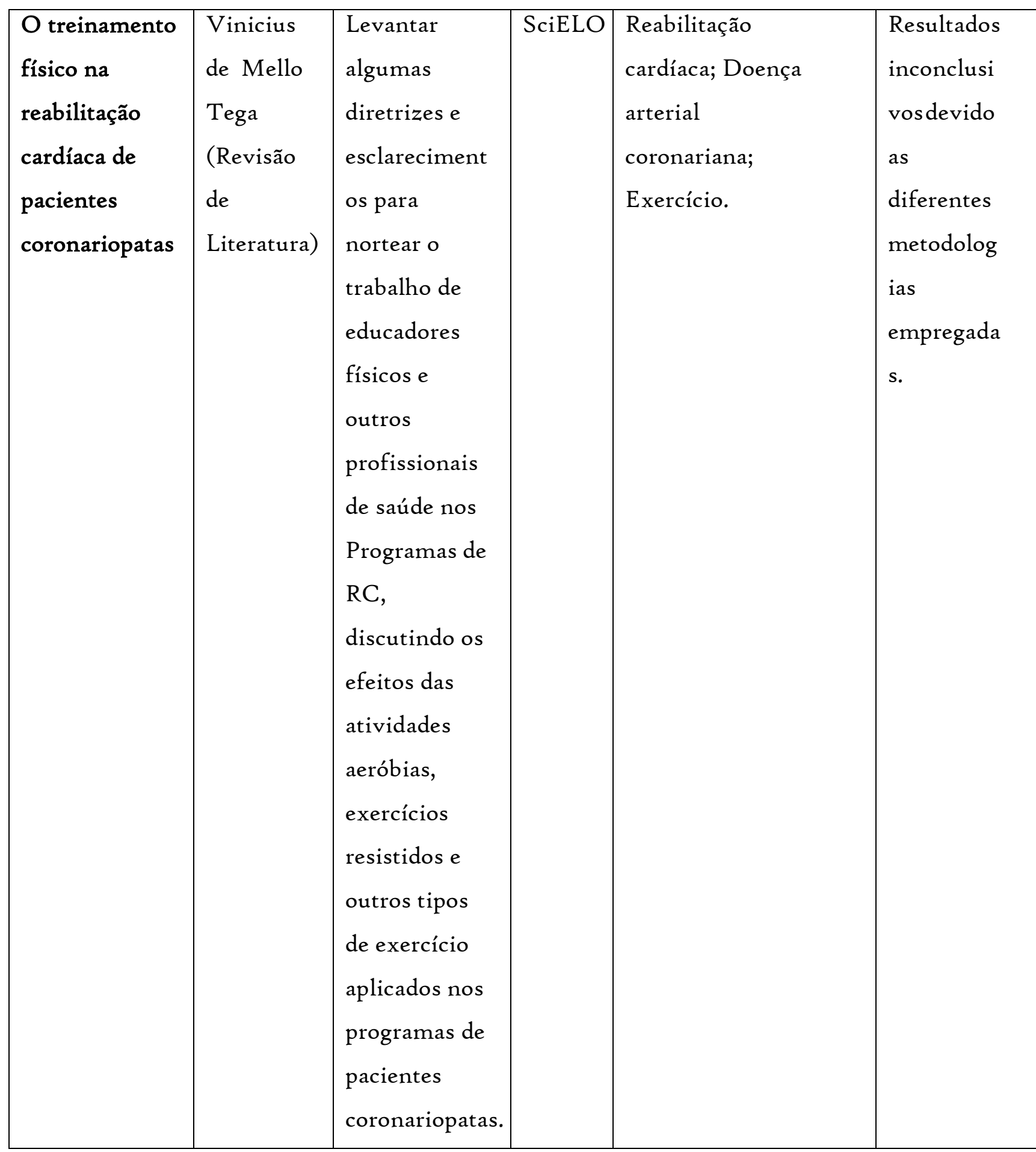




\begin{tabular}{|c|c|c|c|c|c|}
\hline $\begin{array}{l}\text { Prática de } \\
\text { Yogaem } \\
\text { Pacientes com } \\
\text { Insuficiência } \\
\text { Cardíaca } \\
\text { Crônica: uma } \\
\text { meta-análise }\end{array}$ & $\begin{array}{l}\text { Neto et al } \\
\text { (Artigo } \\
\text { de } \\
\text { Revisão } \\
\text { ) }\end{array}$ & $\begin{array}{l}\text { Verificar os } \\
\text { efeitos do } \\
\text { Yoga sobre a } \\
\text { capacidade de } \\
\text { exercício e } \\
\text { qualidade de } \\
\text { vida } \\
\text { relacionada à } \\
\text { saúde } \\
\text { (QVRS) em } \\
\text { pacientes com } \\
\text { ICC. }\end{array}$ & PubMed & $\begin{array}{l}\text { Ioga; Insuficiência } \\
\text { cardíaca; Terapias } \\
\text { Complementares/utiliz } \\
\text { ação; Terapia por } \\
\text { exercício: Metanálise. }\end{array}$ & $\begin{array}{l}\text { Considerand } \\
\text { o osdados } \\
\text { disponíveis, a } \\
\text { presente } \\
\text { meta- análise } \\
\text { indicou que o } \\
\text { yoga } \\
\text { aumenta o } \\
\text { VOz pico e } \\
\text { QVRS em } \\
\text { pacientes } \\
\text { com ICC, e } \\
\text { deve ser } \\
\text { considerado } \\
\text { como um } \\
\text { método }\end{array}$ \\
\hline
\end{tabular}

\begin{tabular}{|l|l|l|l|l|}
\hline & & & & alternativo \\
& & & & de \\
treinament \\
\end{tabular}




\begin{tabular}{|c|c|c|c|c|c|}
\hline $\begin{array}{l}\text { Correlaçã } \\
\text { o entre } \\
\text { força } \\
\text { muscular } \\
\text { respiratóri } \\
\text { a, } \\
\text { capacidade } \\
\text { funcional } \\
\text { e } \\
\text { qualidade } \\
\text { devida na } \\
\text { insuficiên } \\
\text { cia } \\
\text { cardíaca } \\
\text { congestiva }\end{array}$ & $\begin{array}{l}\text { Silva et } \\
\text { al } \\
\text { (Revisão } \\
\text { de } \\
\text { Literatur } \\
\text { a) }\end{array}$ & $\begin{array}{l}\text { Correlaciona } \\
\text { r a qualidade } \\
\text { de vida, } \\
\text { capacidade } \\
\text { funcional e } \\
\text { força } \\
\text { muscular } \\
\text { respiratória } \\
\text { de pacientes } \\
\text { com ICC. }\end{array}$ & $\begin{array}{l}\text { ProQue } \\
\text { st }\end{array}$ & $\begin{array}{l}\text { Insuficiência } \\
\text { cardíaca; } \\
\text { Qualidade de vida; } \\
\text { Músculos } \\
\text { respiratórios; } \\
\text { Capacidade } \\
\text { Funcional. }\end{array}$ & $\begin{array}{l}\text { Conclui-se } \\
\text { que aredução } \\
\text { da força } \\
\text { muscular } \\
\text { respiratória } \\
\text { leva } \\
\text { diminuição } \\
\text { na qualidade } \\
\text { de vida e } \\
\text { piora na } \\
\text { capacidade } \\
\text { funcional. }\end{array}$ \\
\hline $\begin{array}{l}\text { Intervençõe } \\
\text { s não } \\
\text { farmacológi } \\
\text { caspara } \\
\text { melhor } \\
\text { qualidade de } \\
\text { vida na } \\
\text { insuficiênci } \\
\text { a cardíaca: } \\
\text { revisão } \\
\text { integrativa }\end{array}$ & $\begin{array}{l}\text { Amaral et } \\
\text { al } \\
\text { (Revisão } \\
\text { Integrati } \\
\text { va) }\end{array}$ & $\begin{array}{l}\text { Identificar, na } \\
\text { literatura, } \\
\text { artigosque } \\
\text { avaliaram a } \\
\text { efetividade ou } \\
\text { eficácia de } \\
\text { intervenções } \\
\text { não } \\
\text { farmacológica } \\
\text { s para } \\
\text { melhorar a } \\
\text { qualidade de } \\
\text { vidade } \\
\text { pessoas com } \\
\text { insuficiência } \\
\text { cardíaca. }\end{array}$ & $\begin{array}{l}\text { ProQue } \\
\text { st }\end{array}$ & $\begin{array}{l}\text { Estudos de } \\
\text { intervenção; } \\
\text { Insuficiência cardíaca; } \\
\text { Qualidade de vida; } \\
\text { Revisão; Enfermagem. }\end{array}$ & $\begin{array}{l}\text { Estes } \\
\text { resultados } \\
\text { podem } \\
\text { direcionar a } \\
\text { seleção de } \\
\text { intervenções } \\
\text { a serem } \\
\text { implementad } \\
\text { as por } \\
\text { profissionais } \\
\text { de saúde para } \\
\text { identificar as } \\
\text { intervenções } \\
\text { mais eficazes } \\
\text { para } \\
\text { melhorar a } \\
\text { qualidade de }\end{array}$ \\
\hline
\end{tabular}




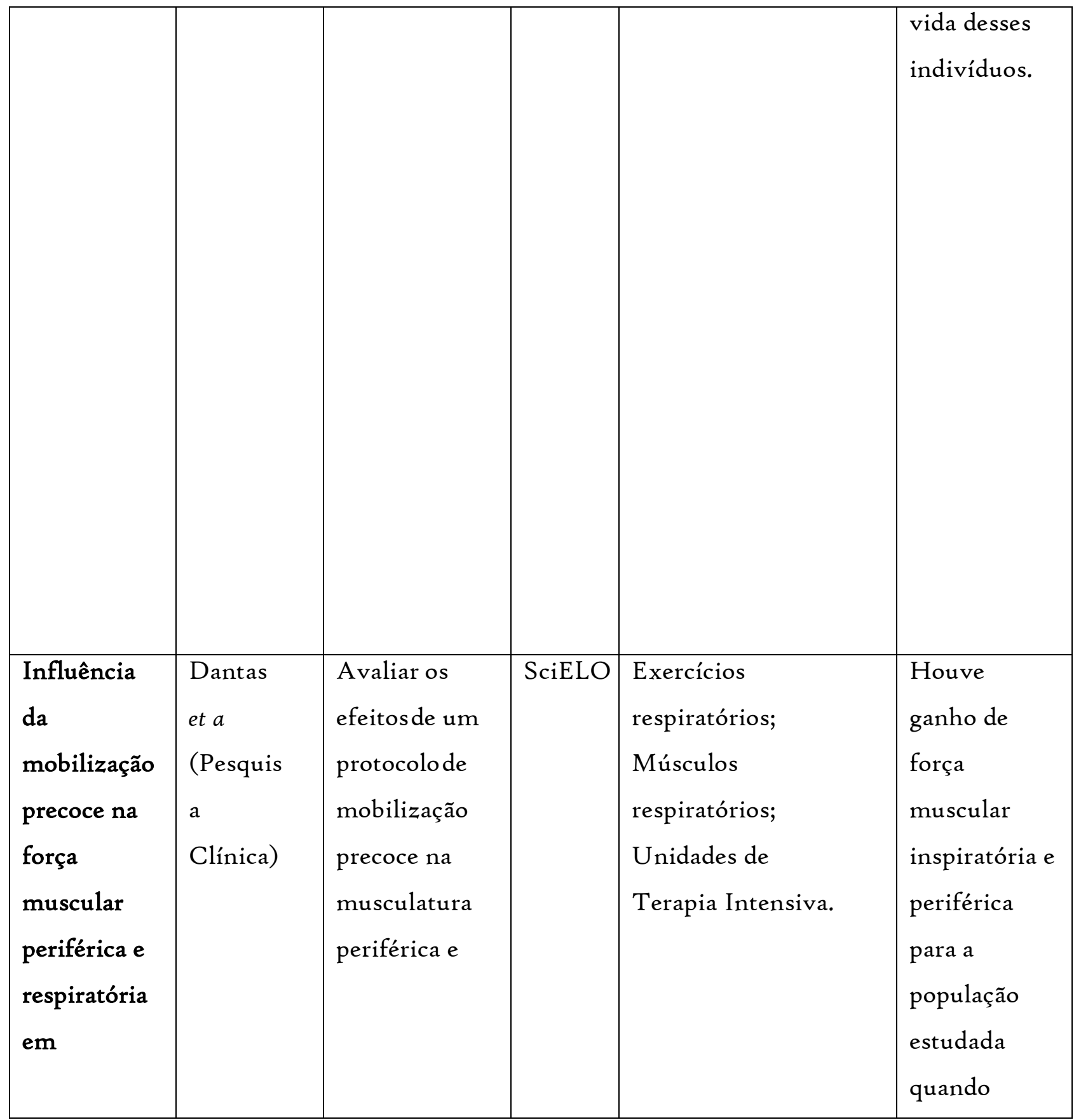

\begin{tabular}{|c|c|c|}
\hline $\begin{array}{l}\text { pacient } \\
\text { es } \\
\text { críticos }\end{array}$ & $\begin{array}{l}\text { respiratória de } \\
\text { pacientes } \\
\text { críticos. }\end{array}$ & $\begin{array}{l}\text { submetida a } \\
\text { umprotocolo } \\
\text { de } \\
\text { mobilização } \\
\text { precoce e } \\
\text { sistematizada. }\end{array}$ \\
\hline
\end{tabular}


Nota: UTI: Unidade de Terapia Intensiva; IC: Insuficiência Cardíaca; CF: Capacidade funcional; RCPM: Reabilitação Cardiopulmonar e Metabólica; QV: Qualidade de vida; VNI: Ventilação não- invasiva; TC6M: Teste de caminhada de seis minutos; VO2: Volume de oxigênio; QVRS: Qualidade de vida relacionada à saúde; ICC: Insuficiência Cardíaca Congestiva.

O repouso delongado no leito deixou de ser uma abordagem seguida pelos fisioterapeutas na Unidade de Terapia Intensiva, pois contribui para o declínio funcional, aumenta os custos assistenciais, redução da qualidade de vida e sobrevida pós-alta, o que promove a inatividade do paciente, ocasionando várias disfunções sistêmicas. Com os avanços tecnológicos e a melhoria do conhecimento científico, aos poucos e com estudos, a compreensão de que a mobilização precoce deveria ser adotada por esses profissionais passou a ser aderida, dando uma nova perspectiva assistencial ao paciente.

A mobilização precoce possui bastante relevância na área da terapia intensiva e nos últimos tempos tem sido explorada e abordada com maior veemência em deliberações que demonstram os efeitos do repouso prolongado no leito. $O$ termo "precoce" se refere às atividades de mobilização quetêm seu início logo após a estabilização do paciente. Dessa forma, este estudo tem por finalidade investigar os efeitos da mobilização precoce em pacientes com IC internados em UTI e, pode-se observar uma resposta positiva procedente desta abordagem.

Um foco multidisciplinar na mobilização precoce é necessário como parte das rotinas clínicasdiárias na UTI. A estrutura da equipe multidisciplinar e a inclusão de fisiatras, médicos, terapeutas ocupacionais, fisioterapeutas, enfermeiros, nutricionistas, fisiologista e assistentes sociais pode servircomo um excelente modelo para a construção de uma equipe de mobilidade precoce na UTI. Isto pode ser útil para avaliar os diferentes componentes de um programa de treinamento próprio, incluindo o tipo, frequência, intensidade e exercícios específicos, além do tipo de programas e intervenções psicossocial ou comportamental utilizados. (BORGES et al, 2009).

As técnicas de mobilização precoce mais utilizadas pelos fisioterapeutas neste setor foram: mobilização passiva, alongamento passivo, posicionamento articular, exercícios ativo assistido e ativo resistido, sentar na cama e/ou poltrona, transferências, cicloergometria para membros inferiores e postura ortostática, transferências, marcha com auxílio evoluindo para marcha sem auxílio, atividades à beira do leito e estimulação elétrica neuromuscular. 
Silva \& Oliveira (2015) demonstraram que tal abordagem resulta em aumento de força muscular periférica, menor número de dias de internação e melhor funcionalidade pós-alta.

Já a imobilização no leito, quando prolongada e associada a outros fatores, pode acarretar em polineuropatia do paciente crítico (PNPC) e síndrome do imobilismo, que são patologias relativamente comuns nas Unidades de Terapia Intensiva (UTI) e configuram grandes desafios para essas unidades (GLAESER et al., 2012; COSTA et al., 2014).

Gosselink et al. (2008) afirmam que mobilização é uma atividade física que seja suficiente para produzir efeitos fisiológicos no paciente, como melhora da ventilação e perfusão, metabolismo muscular, estado de alerta, entre outros.

Ainda segundo Silva \& Oliveira (2015), o efeito mais perceptível da Cinesioterapia na Unidade de Terapia Intensiva é prevenir complicações como a fraqueza muscular, hipotrofia e a síndrome do imobilismo, que consiste em um conjunto de alterações que acometem o indivíduo que se encontra acamado por um longo período de tempo, comprometendo o sistema osteomuscular e, consequentemente, levando a alterações funcionais.

Embora não existam evidências científicas suficientes que atestem a eficácia da cinesioterapia passiva em pacientes internados, esta deve ser iniciada de forma precoce no tratamento desses indivíduos, com o objetivo de manter a amplitude de movimento das articulações, prevenir encurtamento muscular e úlceras de decúbito (FRANÇA et al., 20I2).

O ciclo ergômetro é um instrumento estacionário, que permite rotações cíclicas e pode ser utilizado na execução de exercícios passivos, ativos e ativo assistidos tanto em membros superiores quanto em membros inferiores. Esta é uma técnica que proporciona ao paciente a execução de movimentos repetitivos de baixa resistência com o intuito de promover um treinamento muscular, podendo assim aumentar massa e força muscular, melhorando a eficiência do músculo. Além disso, melhora potencialmente o estado funcional do paciente (GOSSELINK et al., 2008; DANTAS et al., 2012).

Para Almeida e Carvalho (2012), a utilização do cicloergômetro como recurso para o tratamento de pacientes críticos, contribui para a melhora da capacidade funcional, da força muscularperiférica e respiratória, reduzindo a sensação de fadiga e dispneia. Unindo-se a terapia convencional.Dantas et al (2012) fala que há ganho da força muscular inspiratória e periférica para o paciente quando submetido a um protocolo de mobilização precoce e sistematizado. Visto que a mobilização na UTI é viável e segura, pois proporciona redução dos efeitos da imobilidade, buscando a manutenção de sua capacidade funcional e a menor perda das fibras 
musculares que se deterioram com o imobilismo.

Segundo Fonseca et al (2015) a deambulação é uma importante ferramenta terapêutica, segurae que reflete a condição funcional dos pacientes internados na UTI em sua alta. Podendo favorecer a recuperação pós-alta e o retorno às atividades de vida diária o mais precoce possível. Porém, mesmocom significativa melhora, são necessárias mais evidências sobre a sua realização precoce em pacientes de UTI, bem como a relação desta deambulação com os desfechos hospitalares e parâmetros recomendados, como número de passos e qualidade da marcha necessária para caracterizá-la como impactante na melhora clínica dos sujeitos.

Neto et al (2014), alega que o Yoga pode entrar como um tratamento alternativo a pacientes com ICC, pois melhora o $\mathrm{VO}_{2}$ pico e a QVRS. Entrando como um tratamento não farmacológico quepode ser implementado por profissionais de saúde que cuidam de pessoas com IC (AMARAL et al, 2012). Porém, mais estudos são necessários em relação a essa prática em pacientes que estejam em UTI.

Para Oliveira et al (2018) o exercício físico melhora a capacidade funcional em pacientes com insuficiência cardíaca crônica. Entretanto, os efeitos do exercício na IC aguda são desconhecidos.

Ulbrich et al (2013) afirma que a mobilização precoce associada a reabilitação cardiopulmonar e metabólica (RCPM) traz uma melhora na circulação sanguínea periférica, na estrutura muscular esquelética e na capacidade funcional; o retorno precoce às atividades de vida diária (AVD’S); o aumento da capacidade aeróbia; e benefícios no âmbito social. Vale ressaltar que o exercício promove também a melhora da disfunção endotelial e da capacidade oxidativa do músculoesquelético, além de redução da exacerbação neuro-humoral.

De acordo com Borges et al (2009) mais estudos se fazem necessário para se identificar o tipode exercício, duração, intensidade e a repercussão da fisioterapia motora precoce em grupos específicos de pacientes.

\section{CONCLUSÃO}

Após estudo e investigação, conclui-se que a mobilização precoce produz uma resposta positiva na recuperação do paciente. Provocando efeitos benéficos a saúde do paciente com IC.

Os resultados são vários, dentre eles melhoria da função cardiovascular e respiratória, melhora da capacidade funcional e qualidade de vida. Reduzindo o tempo de internação hospitalar. 
Porém, mais estudos devem ser realizados para que haja mais evidências, abordando de forma mais aprofundada a atuação do fisioterapeuta na recuperação de pacientes com IC em UTI, pois há uma escassez de trabalhos com enfoque nesse assunto.

\section{REFERÊNCIAS}

BARRETO, A.C.P; RAMIRES, J.A.P. Insuficiência cardíaca. Arq. Bras. Cardiol. v.7I, n 4. 1998. BORGES, Vanessa Marcos; OLIVEIRA, L. R. C. D; PEIXOTO, Elzo. Fisioterapia motora em pacientes adultos em terapia intensiva. Rev Bras Ter Intensiva, São Paulo, v. 2I, n. 4, p. 446-452, jan./2oro.

YOUTUBE. Mobilização Precoce Revisão Sistemática e Meta-Análise 2019. Disponível em: https://m.youtube.com/watch?v=GYZd6Qiail. Acesso em: 7 mar. 2020.

FONSECA, D. P. et al. Impacto da da deambulação associada à mobilização precoce em pacientes críticos: revisão sistemática. ConScientiae Saúde, Paraná, v. 15, n. 2, p. 325-335, mai./2016.

ULBRICH, A. Z. et al. Capacidade funcional como preditor de qualidade de vida na insuficiência cardíaca. Fisioter. Mov, Curitiba, v. 26, n. 4, p. 845-853, set./2016.

UFTM, HC Da; Fisioterapia no Pós Operatório de Cirurgia Cardíaca no Paciente Adulto: subtítulo do livro. 2.o. ed. Minas Gerais: EBSERH, 20I8. p. I-I9.

\section{INTERFISIO. EFEITOS DA MOBILIZAÇÃO PRECOCE NO PÓS OPERATÓRIO DE} CIRURGIA

CARDÍACA. Disponível em: https://interfisio.com.br/efeitos-da-mobilizacaoprecoce-no-posoperatorio-de-cirurgia-cardiaca/. Acesso em: 15 mar. 2020.

SILVA, Isnanda Tarciara. Efeitos da mobilização precoce em pacientes críticos internados em UTI. Revista Eletrônica da Fainor, Vitória da Conquista, v. 8, n. 2, p. 4I-50, jul./2015.

Comitê Coordenador da Diretriz de Insuficiência Cardíaca. Diretriz Brasileira de Insuficiência 
Cardíaca Crônica e Aguda. Arq Bras Cardiol. 20I8; III(3):436-539 TEGA, V. D. M. O treinamento físico na reabilitação cardíaca de pacientes coronariopatas: subtítulo do artigo. Biblioteca FEF - UNICAMP: subtítulo da revista, Campinas, v. III, n. 3, p. 436-539, dez./2oro.

Amaral DR, Rossi MB, Lopes CT, Lopes JL. Nonpharmacological interventions to improve quality of life in heart failure: an integrative review. Rev Bras Enferm [Internet]. 2017;70(I):18798. DOI: http://dx.doi.org/10.159o/oo34-7167-2016-o112.

SILVA, E. S. D. et al. Correlação entre força muscular respiratória, capacidade funcional e qualidadede vida na insuficiência cardíaca congestiva: subtítulo do artigo. ConScientiae Saúde: subtítulo da revista, Maceió, v. I8, n. 2, p. 149-155, mar./2019.

OLIVEIRA, M. F. et al. Segurança e Eficácia do Treinamento Aeróbio Combinado à Ventilação Não- Invasiva em Pacientes com Insuficiência Cardíaca Aguda: subtítulo do artigo. Arq Bras Cardiol: subtítulo da revista, São Paulo, v. ııo, n. 5, p. 467-475, jan./2or8.

NETO, M. G. et al. Prática de Yoga em Pacientes com Insuficiência Cardíaca Crônica: Uma Meta- análise: subtítulo do artigo. Arq Bras Cardiol.: subtítulo da revista, Salvador, v. Io3, n. 5, p. 433-439,jul./2014.

INTERFISIO. EFEITOS DA MOBILIZAÇÃO PRECOCE NO PÓS OPERATÓRIO DE CIRURGIA

CARDÍACA. Disponível em: https://interfisio.com.br/efeitos-da-mobilizacaoprecoce-no-posoperatorio-de-cirurgia-cardiaca/. Acesso em: 7 abr. 2020.

\section{INTERFISIO. A VIABILIDADE DO USO DO CICLOERGÔMETRO COMO RECURSOTERAPÊUTICO EM PACIENTES INTERNADOS NA UNIDADE DE TERAPIA INTENSIVA:}

REVISÃO DE LITERATURA. Disponível em: https://interfisio.com.br/aviabilidade-douso-do- 
cicloergometro-como-recurso-terapeutico-em-pacientesinternados-na-unidade-de-terapiaintensiva- revisao-de-literatura/. Acesso em: 7 abr. 2020.

INTERFISIO. A ELETROESTIMULAÇÃOCOMO FORMA DE TREINAMENTO EMPACIENTE COM INSUFICIÊNCIA CARDÍACA CONGESTIVA NA UTI.

Disponível em: https://interfisio.com.br/a-eletroestimulacao-como-forma-de-treinamento-empaciente-com-insuficiencia-cardiaca-congestiva-na-uti/. Acesso em: 15 abr. 2020.

INTERFISIO. INSUFICIÊNCIA CARDÍACA: BENEFICIOS DA REABILITAÇÃO CARDÍACA.

Disponível em: https://interfisio.com.br/insuficiencia-cardiaca-beneficiosda-reabilitacaocardiaca/.Acesso em: 15 abr. 2020. 